\title{
Acute and durable effect of inhaled hypertonic saline on mucociliary clearance in adult asthma
}

\author{
William D. Bennett $\mathbb{1}^{1,2}$, Allison Burbank ${ }^{1,3}$, Martha Almond ${ }^{1}$, Jihong Wu ${ }^{1}$, \\ Agathe Ceppe ${ }^{2}$, Michelle Hernandez ${ }^{1,3}$, Richard C. Boucher ${ }^{2}$ and \\ David B. Peden (1) ${ }^{1,3}$
}

Affiliations: ${ }^{1}$ Center for Environmental Medicine, Asthma and Lung Biology, University of North Carolina at Chapel Hill, Chapel Hill, NC, USA. ${ }^{2}$ Pulmonary and Critical Care Medicine, Dept of Medicine, University of North Carolina at Chapel Hill, Chapel Hill, NC, USA. ${ }^{3}$ Division of Allergy and Immunology, Dept of Pediatrics, University of North Carolina at Chapel Hill, Chapel Hill, NC, USA.

Correspondence: William D. Bennett, 104 Mason Farm Road, CB \#7310, Center for Environmental Medicine, Asthma and Lung Biology, University of North Carolina at Chapel Hill, Chapel Hill, NC 27599, USA.

E-mail: William_Bennettlamed.unc.edu

\section{ABSTRACT}

Background: Impaired mucus clearance and airway mucus plugging have been shown to occur in moderate-severe asthma, especially during acute exacerbations. In cystic fibrosis, where airway mucus is dehydrated, it has been shown that inhaled hypertonic saline (HS) produces both acute and sustained enhancement of mucociliary clearance (MCC). The current study was designed to assess the acute and sustained effect of inhaled 7\% HS on MCC in adult asthma.

Methods: Well-controlled, moderate-severe female asthmatic patients $(n=8)$ were screened with a single test dose of albuterol (four puffs by metered-dose inhaler) followed by HS (7\% sodium chloride, $4 \mathrm{~mL}$ using PARI LC Star nebuliser). Spirometry was measured pre-treatment and 5 and 30 min post-treatment for safety. MCC was measured using $\gamma$-scintigraphy on three separate visits: at baseline, during inhalation and $4 \mathrm{~h}$ after a single dose of HS.

Results: MCC was acutely enhanced during HS treatment; mean \pm SD clearance over 60 min of dynamic

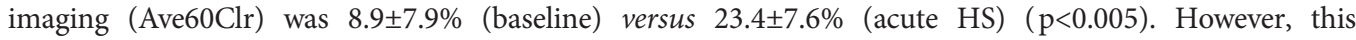
enhancement was not maintained over a 4 -h period where post-HS treatment Ave60Clr was $9.3 \pm 8.2 \%$. In this small cohort we found no decrements in lung function up to $30 \mathrm{~min}$ post-treatment (forced expiratory volume in $1 \mathrm{~s} 97.4 \pm 10.0 \%$ predicted pre-treatment and $98.9 \pm 10.7 \%$ predicted 30 min posttreatment).

Conclusion: While MCC was rapidly enhanced during 7\% HS treatment there was no effect on MCC at $4 \mathrm{~h}$ post-treatment. While these findings may not support aerosolised HS use for maintenance therapy, they do suggest a benefit of treating acute exacerbations in patients with moderate-severe asthma.

@ERSpublications

Acute inhalation of $7 \%$ saline dramatically enhances mucociliary clearance in adult asthmatics, but, unlike in cystic fibrosis, does not provide sustained enhancement $4 \mathrm{~h}$ post-treatment https://bit.ly/2Qiae2K

Cite this article as: Bennett WD, Burbank A, Almond M, et al. Acute and durable effect of inhaled hypertonic saline on mucociliary clearance in adult asthma. ERJ Open Res 2021; 7: 00062-2021 [https://doi.org/10.1183/23120541.00062-2021].

This article has supplementary material available from openres.ersjournals.com

This study is registered at www.ClinicalTrials.gov with identifier number NCT03556683.

Received: 25 Jan 2021 | Accepted: 1 April 2021

Copyright $\odot$ The authors 2021. This version is distributed under the terms of the Creative Commons Attribution NonCommercial Licence 4.0. For commercial reproduction rights and permissions contact permissions@ersnet.org 


\section{Introduction}

Mucociliary clearance (MCC) is a critical innate defence to remove mucus and associated toxins, bacteria, viruses and inflammatory cells/cytokines from the lungs. In vitro studies have demonstrated that well-differentiated airway epithelial cultures lose their ability to transport mucus as they become progressively dehydrated, thus providing a key mechanistic link between mucus dehydration and defective MCC in vivo [1]. Hypertonic saline (HS) has been shown to enhance hydration of mucus and improve mucus rheology in vitro [2-4]. In vivo inhaled HS acutely enhances MCC in healthy volunteers as well as those with asthma and cystic fibrosis (CF) [3, 5-9]. Moreover, in CF the enhancement is both acute and durable, lasting $\geqslant 4 \mathrm{~h}$ with a single dose and $8 \mathrm{~h}$ after repetitive dosing $[3,10]$ and is associated with improved lung function over as little as a 2-week treatment period [3]. However, in healthy subjects the stimulatory effect is acute and paradoxically slowed after $4 \mathrm{~h}$ [7]. Additionally, our recent clinical study in patients with chronic bronchitis showed a general slowing of MCC over a 2-week treatment period [11], and a similar lack of sustained HS effects.

Airway mucus plugging and acute slowing of MCC during exacerbations is a key feature of asthma and contributes to the morbidity and mortality associated with the disease [12-14]. Increased MUC5AC/ MUC5B ratios and associated disulfide bonds in airway mucus [15] as well as ciliary dysfunction [16] are features of severe asthma that probably play a role in reduced MCC. However, there is also evidence for increased dehydration of mucus obtained from patients with asthma. LoughLIN et al. [17] found that induced sputum recovered from asthmatics under baseline (nonexacerbated) conditions had 2.2\% solids compared to $1.6 \%$ in healthy volunteers, with solids percentage being related to neutrophilic content of sputum. While this level of dehydration in asthmatics is less than that observed in CF [18], they do overlap with solids percentage, as we reported in chronic bronchitis [1]. Furthermore, a single treatment with inhaled dry-powder mannitol, a hyperosmolar therapy, in asthmatics showed significant improvement in sputum rheology [19]. These findings support the rationale and potential for the use of inhaled hypertonic saline to treat reduced MCC in asthma.

Because the clinical benefits from inhaled HS probably depend on the duration of this effect on MCC, assessment of MCC at later time points post-HS inhalation will help determine how this treatment may best be applied. In this current open-label pilot study we assessed both the acute (immediate) and durable (4 h post-treatment) efficacy of HS for improving MCC in patients with well-controlled moderate-severe asthma. Finally, due to concerns of airway hyperresponsiveness in these patients, we pre-treated patients with a bronchodilator (albuterol) and monitored lung function before and after HS treatment.

\section{Study design/method}

Study subjects

Adult asthmatics aged 20-53 years with well-controlled moderate-severe asthma at enrolment were studied (defined by step $\geqslant 3$ therapy or by degree of impairment secondary to asthma, per the National Asthma Education and Prevention Program expert panel report [20]). Participants were excluded if forced expiratory volume in $1 \mathrm{~s}\left(\mathrm{FEV}_{1}\right)<70 \%$ predicted without use of short-acting bronchodilator (albuterol) in the previous $8 \mathrm{~h}$. All participants were on a combination long-acting $\beta$-agonist/steroid medication (Advair, Symbicort or Breo) and self-reported adherence to their medication as prescribed during their entire study. All participants were stable, i.e. no signs of exacerbation, when studied.

\section{Study design}

Participants had a baseline MCC assessment by $\gamma$-scintigraphy imaging over a 2 -h period following inhalation of radiolabelled particles (technetium-99m sulfur colloid) as described in detail previously [7, 21] (figure 1, supplementary videos). At the conclusion of the 2-h baseline MCC assessment subjects were screened for bronchial hyperreactivity associated with albuterol/HS treatment. $15 \mathrm{~min}$ after pre-treatment with four puffs of albuterol $(90 \mu \mathrm{g}$ each via metered-dose inhaler (MDI) with spacer), subjects received a screening dose of HS inhalation by PARI LC Star nebuliser ( $4 \mathrm{~mL} 7 \%$ sodium chloride inhaled until sputter). Spirometry was measured at 5 and $30 \mathrm{~min}$ after completion of HS inhalation. Participants with a 10\% fall in $\mathrm{FEV}_{1}$ that persisted for $\geqslant 30$ min were excluded from further study.

At least 1 week after the screening/baseline MCC visit, we assessed the acute effects of HS on MCC (figure 1). Immediately following the initial 4-min deposition scan of the MCC dynamic imaging, subjects received albuterol and 7\% HS inhalation over a 25-min period (as described earlier for screening visit) while being scanned for MCC through the 2-h period (figure 1). Cough frequency was monitored during the measure of MCC. After $\geqslant 1$ week, participants returned to assess the durability of albuterol/HS on MCC. Subjects again received albuterol pre-treatment and HS inhalation as described earlier, but the radioaerosol inhalation and MCC measurement began $4 \mathrm{~h}$ later. For each MCC measurement, subjects returned the following day for a 30 -min image to assess retention at $24 \pm 4 \mathrm{~h}$ after initial deposition. 
Baseline MCC

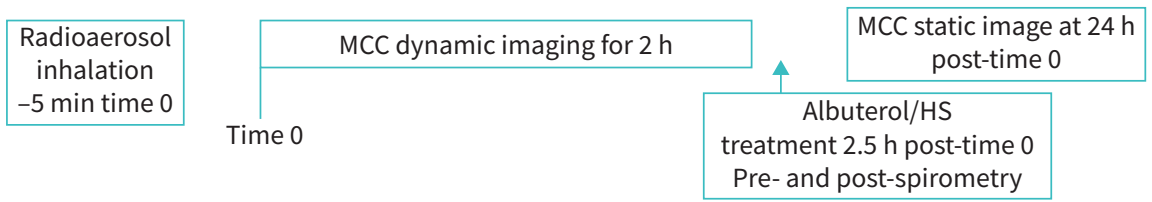

Acute HS treatment MCC

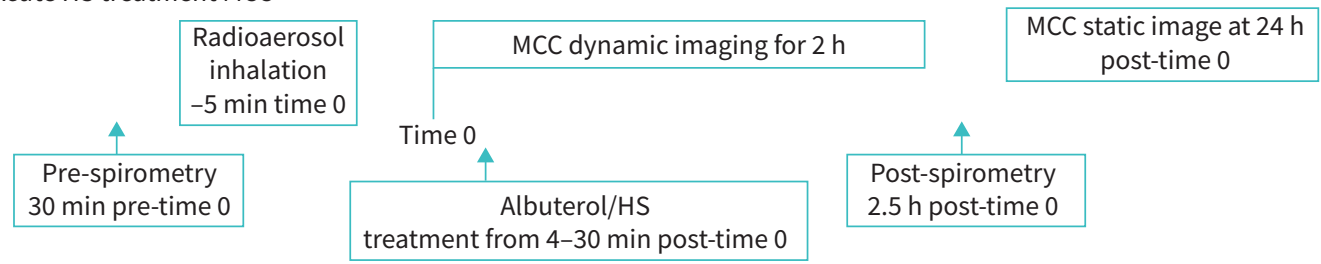

Post-4-h HS treatment MCC

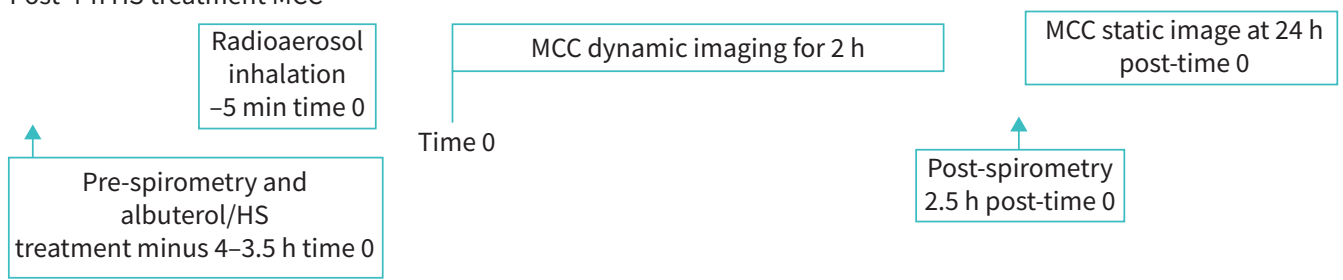

FIGURE 1 Study design. MCC: mucociliary clearance; HS: hypertonic saline.

Baseline MCC measured in a control group of healthy non-asthmatic female adults ( $\mathrm{n}=19$ ) who participated in previous $[7,21]$ and current studies were included for comparison to baseline MCC in the asthmatic adults.

\section{MCC analysis}

By computer analysis, a rectangular whole and central region was circumscribed over the right lung defined by a cobalt-57 transmission scan in each subject [7]. The region was used to determine whole-lung retention (Rt) (decay- and background-corrected) as a fraction of the initial counts over the 2-h imaging period. To describe each retention versus time dataset (e.g. mean data shown in figure 2), the

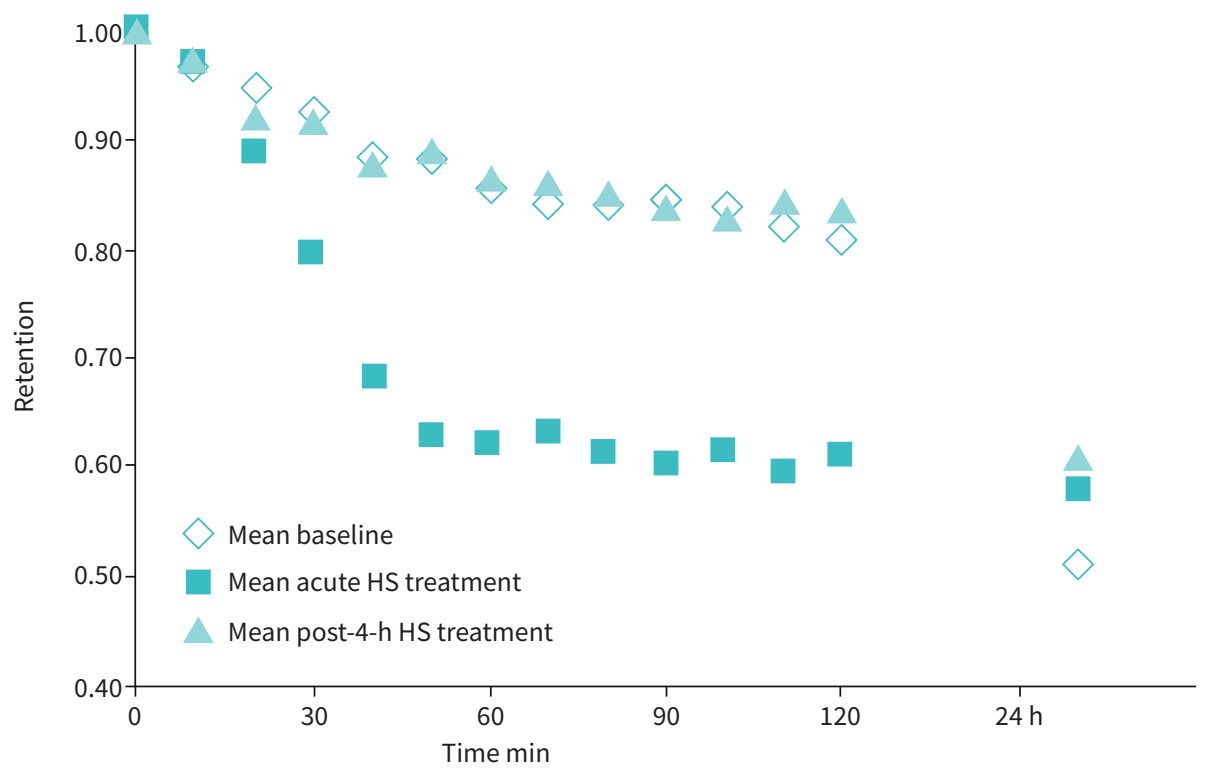

FIGURE 2 Mean retention versus time for the baseline, acute hypertonic saline (HS) treatment, and post-4-h HS treatment for eight adult female asthmatics. 
average percentage clearance (or $100 \times(1-\mathrm{Rt})$ ) over the 60 - and 120 -min period of observation were computed (i.e. average of the 10 -min clearance values from 10 to 60 or $120 \mathrm{~min}$ ). These computed values are signified as Ave60Clr and Ave120Clr, respectively. This method for characterising nonlinear retention versus time data over a given period (figure 2) allows for all 10-min datapoints to be considered, in our case area under the curve expressed in terms of average clearance $[22,23]$ over that period. Regional deposition, a potential confounder of MCC measurements, was also determined by central/peripheral (C/P) ratio for each measure of MCC. Increasing $\mathrm{C} / \mathrm{P}$ is associated with an increase in average clearance $[22,23]$. The retention at $24 \mathrm{~h}$ was compared between study visits (figure 2) as an index of initial, regional deposition of radioaerosol between bronchial and alveolated airways [22].

MCC in the healthy control group was only compared through 60-min imaging (i.e. Ave60Clr) due to interventions post-60 min in some of these healthy control studies.

\section{Statistics}

The pre-specified primary study outcome was whole lung AveClr60 between baseline versus acute versus 4-h post-challenge visits. Secondary outcomes included changes in spirometry and other MCC outcomes (AveClr120, C/P, 24-h retention). From previous studies we have seen that the distribution of MCC outcomes is normal $[3,7,11]$; we did not expect strong outliers even under the conditions of low numbers.

Data were analysed using a repeated-measure ANOVA using visit as the primary fixed factor (baseline, acute HS, durability HS). Multiple comparisons between visits were then adjusted using Tukey. Effects of $\mathrm{C} / \mathrm{P}$ and $\mathrm{FEV}_{1}$ were analysed by adding them as covariates in the model. The relationship between specific outcomes were determined by Pearson's correlations at each visit. Comparisons between asthmatic and healthy subjects were made with independent t-tests. p-values $<0.05$ were considered significant. All statistical analyses were conducted utilising SAS v9.4 and JMP Pro 14 (SAS, Cary, NC, USA) statistical software.

\section{Study approval}

The study was approved by the University of North Carolina institutional review board, and subjects provided written informed consent. The study was registered at Clinical Trials.gov (NCT03556683).

\section{Results}

Nine (eight females, one male) asthmatic patients completed the study as designed. Due to the low number of males recruited to the study before interruption by the coronavirus disease 2019 pandemic, we narrowed our findings reported here to female subjects $(n=8)$ including MCC comparison to healthy nonasthmatic females $(n=19)$. No participants were excluded based on the HS screen. Mean \pm SD age was $29.6 \pm 12.1$ years and $27.7 \pm 6.6$ years for asthmatics and nonasthmatics, respectively. On the screening study day, the $\mathrm{FEV}_{1}$ normalised as a percentage of the predicted $\mathrm{FEV}_{1}$ value was $97.4 \pm 10.0 \%$ pre-HS treatment and $97.5 \pm 12.2 \%$ and $98.9 \pm 10.7 \%$ at 5 and $30 \mathrm{~min}$ post-HS treatment, respectively (nonsignificant).

Figure 2 shows the significantly different mean retention in time data for the three MCC measurements in the female asthmatics $(\mathrm{p}=0.0017)$. The acute challenge with HS produced an immediate, rapid clearance of the radiolabelled particles compared to baseline (see supplementary videos), Ave60Clr 8.9 $\pm 7.9 \%$ (baseline) versus $23.4 \pm 7.6 \%$ (acute HS) $(\mathrm{p}<0.005)$. In addition, over the entire 120 -min period of MCC measurement the average clearance was different between the three MCC measures; greater for acute HS compared to baseline, Ave120Clr $12.8 \pm 8.8 \%$ (baseline) versus $31.1 \pm 8.6 \%$ (acute HS) $(\mathrm{p}<0.002$ ). It was evident that only a small fraction of particles were still available for clearance between $60 \mathrm{~min}$ and $24 \mathrm{~h}$ for the acute HS case. By contrast, there was no effect of HS on MCC measured $4 \mathrm{~h}$ post-challenge compared to baseline, Ave60Clr $9.3 \pm 8.2 \%$ and Ave120Clr $12.4 \pm 8.6 \%$ for $4 \mathrm{~h}$ post-challenge. The 24 -h retention was not different between the baseline, acute HS and 4 h post-HS study visits $(p=0.14) ; 0.51 \pm 0.06,0.58 \pm 0.09$ and $0.61 \pm 0.13$, respectively.

Regional deposition $(\mathrm{C} / \mathrm{P})$ was not different between the three MCC measurements: C/P $2.2 \pm 0.5$ (baseline), $2.1 \pm 0.4$ (acute HS) and $2.1 \pm 0.4$ (4 h post-HS). Both Ave60Clr and Ave120Clr were correlated with $\mathrm{C} / \mathrm{P}$ for the baseline MCC measurements $(\mathrm{R}=-0.84, \mathrm{p}<0.01$ and $\mathrm{R}=-0.87, \mathrm{p}<0.01$, respectively). Pre-FEV 1 was not a significant covariate for either Ave60Clr or Ave120Clr. Spontaneous cough only occurred for the acute HS challenge in six of the eight asthmatics during the MCC measurement and was minimal (four or fewer coughs for any subject, mean coughs 2.3 for all).

Baseline MCC trended slower in the small group of female asthmatics compared to the nonasthmatic female cohort, Ave60Clr 8.9 \pm 7.9 versus $13.9 \pm 6.5 \%$ ( $\mathrm{p}=0.09$ ), respectively, while neither C/P $2.2 \pm 0.2$ nor 24-h retention $0.51 \pm 0.10$ for healthy nonasthmatics was different from the asthmatics. 


\section{Discussion}

As expected, the acute inhalation of HS in these female asthmatics enhanced MCC compared to baseline. The immediate and very rapid effect was somewhat unexpected, especially without the accompanied coached coughing that is included in HS inhalation/sputum induction protocols [24, 25]. Despite this dramatic acute effect, MCC was unaffected $4 \mathrm{~h}$ post-treatment relative to baseline. This is in contrast to findings in CF, where we found enhanced MCC at $4 \mathrm{~h}$ post-HS inhalation, similar to that observed acutely relative to baseline [10]. Unlike healthy volunteers, MCC (Ave60Clr and Ave120Clr) was unchanged between baseline and $4 \mathrm{~h}$ post-treatment, suggesting that the resident mucus had not been depleted (compromised) by the acute HS treatment $4 \mathrm{~h}$ earlier [7], at least in the larger bronchial airways. Our recent study on the effects of HS treatment in chronic bronchitis showed slowing of MCC relative to baseline after multiple treatments over a 2-week period [11].

Whether a longer-term effect may also occur in asthma is uncertain. It may also be that enhanced MCC could have been observed at earlier times post-treatment (e.g. $2 \mathrm{~h})$. The dramatic, acute effect may be beneficial where acute mucus clearance is needed, e.g. acute exacerbations, where there may be severe slowing of MCC $[13,14]$ and where in-patient treatment will allow monitoring of any HS-induced hyperreactivity. This is analogous to the indications for use of established, quick-relief medications for asthma (such as albuterol). While the absence of a durable HS effect in these asthmatics may not support its use for maintenance therapy, it may still be useful for asthmatics in whom periodic clearance of mucus may be beneficial. It will be important to consider the safety and duration of effect of HS when exploring potential uses for asthma.

We included MDI albuterol treatment prior to all HS treatment in the current study to mitigate potential airway hyperreactivity in these participants. Therefore, it should be emphasised that any treatment effects we observed on MCC were for the combination of MDI albuterol followed by nebulised HS. $\beta$-agonists delivered by MDI have been shown to acutely enhance MCC in asthma [26, 27], but not to the extent observed in the present study. In fact, in one case, no stimulation of MCC was observed despite the fact that delivery of terbutaline by MDI produced significant bronchodilatation [28]. These studies would suggest that a greater dose of $\beta$-agonist is required for enhancement of MCC than is needed to induce bronchodilation. Our study design did not allow for distinguishing the relative contribution of HS versus albuterol on MCC, but it is clear that any HS treatment will always include pre-treatment with a short-acting bronchodilator to mitigate any HS-induced hyperreactivity.

There was also some very infrequent, spontaneous cough (see supplementary data) associated with the acute treatment; average 2.3 coughs for all. In previous studies in which we incorporated 60 voluntary coughs during the 60-90 min period of MCC measures in healthy adults [23] we found a 70\% increase in clearance for MCC versus MCC+cough, much less than observed here over the first 60 min (Ave60Clr), a $163 \%$ enhancement with only a few coughs. The individual data (supplementary material) showed that even those with no spontaneous coughing had a significant enhancement of MCC with albuterol/HS. While we cannot quantify the contribution of a few spontaneous coughs on clearance in the current study, it nevertheless can be argued that some spontaneous cough associated with the albuterol/HS treatment is advantageous if it aids in the clearance of mucus from the airways.

The single treatment of inhaled 7\% HS in our study of adult females with moderate-severe asthma produced no evidence of airway hyperreactivity, e.g. coughing or acute reduction in lung function. Retrospective analysis of induced sputum procedures in mild-moderate asthmatics (successive inhalations of $3 \%, 4 \%$ and $5 \%$ HS) also showed no adverse effects associated with HS challenge [24]. Nevertheless, the small number of participants in the current study does not allow for adequate power to exclude potential adverse effects from inhaled 7\% HS. It may be that a lower tonicity of saline (e.g. 3\%) would prove to be as effective for acutely enhancing MCC while providing a greater margin of safety compared to $7 \%$ HS. Finally, it is not clear whether multiple HS treatments would be well tolerated, but our experience with multiple induced sputum procedures in asthmatics has shown no HS-associated adverse events.

Finally, this pilot study was limited to female adults. While there is some suggestion that healthy males may exhibit slower baseline MCC than females [23], there are no data to suggest gender-based differences for MCC in asthmatics nor differences in HS responsiveness. Therefore, we think it unlikely that further addition of males to the study would change the fundamental findings we observed and report here in females.

\section{Conclusion}

MCC was dramatically enhanced during and immediately after acute treatment with inhaled 7\% HS delivered by PARI LC Star nebuliser, but had no prolonged effect at $4 \mathrm{~h}$ post-treatment. Due to the immediate and short-lived effect of HS on MCC in asthma, we hypothesise that this intervention may be most effective for periodic clearance of mucus or with viral or allergen-induced acute exacerbation where 
acute mucus slowing/plugging may be present. Future studies to assess the effect and safety of HS treatment on experimental challenges [14] in these patients may provide support for moving forward with such a treatment regimen.

Support statement: This study was supported by National Institutes of Health grant P01 HL108808. Funding information for this article has been deposited with the Crossref Funder Registry.

Conflict of interest: W.D. Bennett reports grants from the NIH during the conduct of the study. A. Burbank reports grants from the NIH during the conduct of the study. M. Almond reports grants from the NIH during the conduct of the study. J. Wu reports grants from the NIH during the conduct of the study. A. Ceppe reports grants from the NIH during the conduct of the study. M. Hernandez reports grants from NHLBI during the conduct of the study and personal fees for consultation on asthma therapies from GSK outside the submitted work. R.C. Boucher reports grants from the NIH; and personal fees from Parion Sciences, a privately held UNC spin-out company focused on developing therapies for CF, both during the conduct of the study. He is Chairman of the Board of Parion, and has equity in it and receives monetary compensation as Board Chair. D.B. Peden reports grants from the NIH during the conduct of the study and from the US Environmental Protection Agency outside the submitted work.

\section{References}

1 Anderson WH, Coakley RD, Button B, et al. The relationship of mucus concentration (hydration) to mucus osmotic pressure and transport in chronic bronchitis. Am J Respir Crit Care Med 2015; 192: 182-190.

2 Goralski JL, Wu D, Thelin WR, et al. The in vitro effect of nebulised hypertonic saline on human bronchial epithelium. Eur Respir J 2018; 51: 1702652.

3 Donaldson SH, Bennett WD, Zeman KL, et al. Mucus clearance and lung function in cystic fibrosis with hypertonic saline. N Engl J Med 2006; 354: 241-250.

4 King M, Dasgupta B, Tomkiewicz RP, et al. Rheology of cystic fibrosis sputum after in vitro treatment with hypertonic saline alone and in combination with recombinant human deoxyribonuclease I. Am J Respir Crit Care Med 1997; 156: 173-177.

5 Pavia D, Thomson ML, Clarke SW. Enhanced clearance of secretions from the human lung after the administration of hypertonic saline aerosol. Am Rev Respir Dis 1978; 117: 199-203.

6 Sood N, Bennett WD, Zeman K, et al. Increasing concentration of inhaled saline with or without amiloride: effect on mucociliary clearance in normal subjects. Am J Respir Crit Care Med 2003; 167: 158-163.

7 Bennett WD, Wu J, Fuller F, et al. Duration of action of hypertonic saline on mucociliary clearance in the normal lung. J Appl Physiol 2015; 118: 1483-1490.

8 Daviskas E, Anderson SD, Gonda I, et al. Inhalation of hypertonic saline aerosol enhances mucociliary clearance in asthmatic and healthy subjects. Eur Respir J 1996; 9: 725-732.

9 Robinson M, Hemming AL, Regnis JA, et al. Effect of increasing doses of hypertonic saline on mucociliary clearance in patients with cystic fibrosis. Thorax 1997; 52: 900-903.

10 Trimble AT, Whitney Brown A, Laube BL, et al. Hypertonic saline has a prolonged effect on mucociliary clearance in adults with cystic fibrosis. J Cyst Fibrosis 2018; 17: 650-656.

11 Bennett WD, Henderson AG, Ceppe A, et al. Effect of hypertonic saline on mucociliary clearance and clinical outcomes in chronic bronchitis. ERJ Open Res 2020; 6: 00269-2020.

12 Kuyper LM, Paré PD, Hogg JC, et al. Characterization of airway plugging in fatal asthma. Am J Med 2003; 115 6-11.

13 Messina, MS, O'Riordan TG, Smaldone GC. Changes in mucociliary clearance during acute exacerbations of asthma. Am Rev Respir Dis 1991; 143: 993-997.

14 Bennett WD, Herbst M, Alexis NE, et al. Effect of inhaled dust mite allergen on regional particle deposition and mucociliary clearance in allergic asthmatics. Clin Exp Allergy 2011; 41: 1719-1728.

15 Fahy JV, Dickey BF. Airway mucus function and dysfunction. N Engl J Med 2010; 363: 2233-2247.

16 Biju T, Rutman A, Hirst RA, et al. Ciliary dysfunction and ultrastructural abnormalities are features of severe asthma. J Allergy Clin Immunol 2010; 126: 722-729.

17 Loughlin CE, Esther CR Jr, Lazarowski ER, et al. Neutrophilic inflammation is associated with altered airway hydration in stable asthmatics. Respir Med 2010; 104: 29-33.

18 Henderson AG, Ehre C, Button B, et al. Cystic fibrosis airway secretions exhibit mucin hyperconcentration and increased osmotic pressure. J Clin Invest 2014; 124: 3047-3060.

19 Daviskas E, Anderson SD, Young IH. Inhaled mannitol changes the sputum properties in asthmatics with mucus hypersecretion. Respirology 2007; 12: 683-691.

20 National Heart, Lung, and Blood Institute National Asthma Education and Prevention Program. Expert Panel Report 3: Guidelines for the Diagnosis and Management of Asthma. 2007. Available from: www.nhlbi.nih.gov/ health-topics/guidelines-for-diagnosis-management-of-asthma/

21 Zeman $\mathrm{KL}, \mathrm{Wu}$ J, Bennett WD. Targeting aerosolized drugs to the conducting airways using very large particles and extremely slow inhalations. J Aerosol Med Pulm Drug Deliv 2010; 23: 363-369.

22 Bennett WD, Daviskas E, Hasani A, et al. Mucociliary and cough clearance as a biomarker for therapeutic development. J Aerosol Med Pulm Drug Deliv 2010; 23: 261-272.

23 Bennett WD, Laube BL, Corcoran T, et al. Multisite comparison of mucociliary and cough clearance measures using standardized methods. J Aerosol Med Pulm Drug Deliv 2013; 26: 157-164.

24 Alexis NE, Bennett W, Peden DB. Safety and benefits of inhaled hypertonic saline following airway challenges with endotoxin and allergen in asthmatics. J Asthma 2017; 54: 957-960.

25 Alexis NE, Hu SC, Zeman K, et al. Induced sputum derives from the central airways: confirmation using a radiolabeled aerosol bolus delivery technique. Am J Respir Crit Care Med 2001; 164: 1964-1970.

26 Mortensen J, Groth S, Lange P, et al. Effect of terbutaline on mucociliary clearance in asthmatic and healthy subjects after inhalation from a pressurised inhaler and a dry powder inhaler. Thorax 1991; 46: 817-823. 
27 Pavia D, Agnew JE, Sutton PP, et al. Effect of terbutaline administered from metered dose inhaler (2 mg) and subcutaneously $(0.25 \mathrm{mg})$ on tracheobronchial clearance in mild asthma. $\mathrm{Br} J$ Dis Chest 1987; 81: 361-370.

28 Bateman JR, Pavia D, Sheahan NF, et al. Effects of terbutaline sulphate aerosol on bronchodilator response and lung mucociliary clearance in patients with mild stable asthma. Br J Clin Pharmacol 1983; 15: 695-700. 